\title{
Impact of enzalutamide on patient-related outcomes in metastatic castration-resistant prostate cancer: current perspectives
}

This article was published in the following Dove Press journal:

Research and Reports in Urology

29 November 2016

Number of times this article has been viewed

Jia Luo'

Julie N Graff ${ }^{2,3}$

'Department of Medicine; ${ }^{2}$ Knight Cancer Institute, Oregon Health

\& Science University, ${ }^{3} \mathrm{VA}$ Portland Health Care System, Portland,

OR, USA
Correspondence: Julie N Graff

Knight Cancer Institute, 3303 SW Bond Ave, MC CHI4R, Portland, OR 97239,

USA

Email graffj@ohsu.edu
Abstract: Prostate cancer claims the lives of more than 25,000 men in the United States yearly, most from metastatic disease. In the past decade, several new medications have been approved for the treatment of metastatic prostate cancer including the antiandrogen enzalutamide. In addition, there has been mounting interest in evaluating health-related quality of life (QoL) in patients with cancer including new more detailed recommendations released by the Prostate Cancer Working Group 3 on how to evaluate patient-related outcomes in clinical trials. A total of four randomized double-blind placebo-controlled trials have evaluated patients with metastatic castration-resistant prostate cancer (mCRPC) randomized to receive enzalutamide vs control or bicalutamide. Each study used validated health-related QoL and pain surveys to evaluate patient-related outcomes. The studies suggest that patients with mCRPC, including those aged 75 years and older, have favorable overall QoL scores taking enzalutamide compared to standard of care. There was short-term improved pain control in patients taking enzalutamide compared to those in the placebo group. Some commonly reported adverse effects included fatigue, back pain, and hot flashes. These studies were limited in their patient attrition in filling out surveys as well as difficulty in comparing them to each other. Future studies examining patients with mCRPC taking enzalutamide will have to rigorously standardize ways patient-reported outcomes are collected and evaluate patients in a more diversified real-world population.

Keywords: enzalutamide, metastatic castration-resistant prostate cancer, patient-related outcomes

\section{Introduction}

The incidence of prostate cancer is about 180,000 new cases per year in the United States and approximately 1.1 million new cases per year globally. ${ }^{1,2}$ Prostate cancer is the most commonly diagnosed cancer in men and accounts for about $8 \%$ of cancer deaths in the USA. ${ }^{1}$ Most of these deaths are from those with metastatic disease.

The treatment of metastatic prostate cancer has evolved rapidly. After the discovery of the androgen dependence of this cancer in the 1940s followed by the androgen receptor itself in the 1960s, the 1980-1990s saw the development of luteinizing hormonereleasing hormone antagonists and the first oral antiandrogens flutamide, nilutamide, and bicalutamide. ${ }^{3}$ The cornerstone of therapy for advanced prostate cancer continues to be growth inhibition through androgen blockade leading to apoptosis of cancer cells. First-line treatments target a serum testosterone level of $<50 \mathrm{ng} / \mathrm{dL}$, termed castration. Trials have continued to explore the benefits of castration therapy vs combined androgen blockade with oral antiandrogens added to luteinizing hormone-releasing hormone antagonists. ${ }^{4,5}$ Prostate cancer eventually becomes resistant to castration, and an early 
marker is a rising serum prostate-specific antigen (PSA) despite serum testosterone levels $<50 \mathrm{ng} / \mathrm{dL}$. PSA progression is defined by the Prostate Cancer Working Group 3 as a $25 \%$ increase and a minimum rise of $2 \mathrm{ng} / \mathrm{mL}$ in PSA above the nadir, confirmed with a second value 3 or more weeks later. ${ }^{6}$ Despite being termed castrate resistant, castrationresistant prostate cancer (CRPC) continues to be dependent on androgen receptor signaling, which led to the development of new second-generation antiandrogens. Recently approved agents for the metastatic castration resistant setting include enzalutamide and abiraterone, with several ongoing clinical trials for additional agents. ${ }^{7,8}$ The current National Comprehensive Cancer Network guidelines for metastatic CRPC recommend treatment with either hormone therapy, chemotherapy, enzalutamide, or abiraterone with prednisone, or pursue clinical trial. ${ }^{9}$ Enzalutamide (MDV3100) is a second-generation antiandrogen that binds the androgen receptor at its ligand-binding domain, inhibiting its activation, and may be more potent than its first-generation predecessors. ${ }^{10,11}$ It is dosed at $160 \mathrm{mg}$ oral daily with no hepatic or renal adjustment, although the dosing for a creatinine clearance of $<30 \mathrm{~mL} / \mathrm{min}$ is not defined. ${ }^{12}$

Patient-reported outcomes (PROs) in oncology are increasingly being evaluated for in clinical trials because clinicians have been found to underreport symptoms and PROs have been found to impact disease trajectory. ${ }^{13} \mathrm{~A}$ single-center randomized study of 776 patients from 2007 to 2011 found that routine assessments of PROs led to improved quality of life $(\mathrm{QoL}) .{ }^{14}$ In this study, patients receiving chemotherapy for advanced solid tumors were randomized to reporting 12 symptoms via tablets vs usual care. E-mail alerts were sent to nurses if the symptoms worsened, and printouts were given to oncologists during the clinic visits. The primary outcome was a clinically meaningful improvement in the validated EQ-5D survey, with a 6-point decline being clinically meaningful. Patients had similar baseline characteristics. The results showed a median decline of 7.1 points in the usual care group as compared to a 1.4-point decline in the symptom reporting group $(p<0.001)$. In addition, there were fewer emergency room visits ( $34 \%$ vs $41 \%$, $p=0.02$ ), and patients remained on chemotherapy longer (mean: 8.2 months vs 6.3 months, $p=0.002$ ).

Given the importance of identifying PROs, this review seeks to highlight the PROs of individuals taking enzalutamide in an effort to guide practitioners who will be prescribing and patients who will be taking this drug.

\section{Methods}

A PubMed literature search was performed to look for randomized controlled trials of enzalutamide compared to placebo or standard of care in the treatment of advanced prostate cancer, and for additional papers related to these trials reporting on PROs. The decision was made to only report randomized double-blind controlled studies involving enzalutamide to minimize confounding and placebo effects. Boolean search terms included enzalutamide, MDV3100, "metastatic castration resistant prostate cancer", "metastatic prostate cancer", and "castration resistant prostate cancer". This is a narrative overview of the literature that was found.

\section{Results}

\section{Summary of trials}

There were four trials examining enzalutamide compared to placebo or bicalutamide in patients with advanced prostate cancer: AFFIRM (2012), PREVAIL (2014), STRIVE (2016), and TERRAIN (2016) (Table 1).

AFFIRM was a Phase III international randomized double-blind study in patients with metastatic CRPC (mCRPC) who had prior chemotherapy. ${ }^{15}$ The trial enrolled 1,199 patients between September 2009 and November 2010 and randomized them in a 2:1 ratio to receive enzalutamide vs placebo. The primary end point was overall survival (OS),

Table I Overview of the randomized double-blinded controlled trials examining enzalutamide and the patient-related outcome measures used

\begin{tabular}{|c|c|c|c|c|c|}
\hline Trial & Arms & Study design & $\mathbf{n}$ & Primary outcome & PRO instruments used \\
\hline AFFIRM ${ }^{15}$ & $\begin{array}{l}\text { Enzalutamide vs placebo (mCRPC } \\
\text { post-chemo) }\end{array}$ & Phase III & 1,199 & $\begin{array}{l}\text { OS; mOS I } 8.4 \text { months vs I } 3.6 \text { months } \\
\text { (HR: } 0.63, p<0.00 I \text { ) }\end{array}$ & FACT-P, BPI-SF \\
\hline PREVAIL ${ }^{16}$ & $\begin{array}{l}\text { Enzalutamide vs placebo (mCRPC } \\
\text { pre-chemo) }\end{array}$ & Phase III & ।,7। & $\begin{array}{l}\text { OS, radiographic PFS; deaths } 28 \% \text { vs } 35 \% \\
\text { (HR: } 0.7 \mathrm{I}, p<0.00 \mathrm{I} \text { ) }\end{array}$ & FACT-P, EQ-5D, BPI-SF \\
\hline STRIVE ${ }^{17}$ & $\begin{array}{l}\text { Enzalutamide vs bicalutamide } \\
\text { (CRPC) }\end{array}$ & Phase II & 396 & $\begin{array}{l}\text { PFS; in mCRPC I } 6.5 \text { vs } 5.5 \text { months (HR: } 0.24 \text {, } \\
p<0.00 I \text { ) }\end{array}$ & FACT-P \\
\hline TERRAIN 18 & $\begin{array}{l}\text { Enzalutamide vs bicalutamide } \\
\text { (mCRPC) }\end{array}$ & Phase III & 375 & PFS; I 5.7 vs 5.8 months (HR: $0.44, p<0.000$ I) & FACT-P, BPI-SF \\
\hline
\end{tabular}

Abbreviations: PRO, patient-related outcome; CRPC, castration-resistant prostate cancer; mCRPC, metastatic castration-resistant prostate cancer; FACT-P, Functional Assessment of Cancer Therapy-Prostate; OS, overall survival; HR, hazard ratio; PFS, progression-free survival; BPI-SF, Brief Pain Inventory - Short Form; mOS, median overall survival. 
defined as time from randomization to death from any cause. QoL measures were a secondary end point. The trial stopped after a planned interim analysis demonstrated improved OS with enzalutamide, with a median OS of 18.4 months in the enzalutamide group compared to a median OS of 13.6 months in the placebo group (hazard ratio [HR]: $0.63,95 \%$ confidence interval [CI]: [0.53-0.75]; $p<0.001)$.

PREVAIL was a Phase III trial that enrolled 1,717 patients with mCRPC who had not received chemotherapy and randomized them in a 1:1 ratio to receive enzalutamide or placebo. ${ }^{16}$ The trial recruited patients globally between September 2010 and September 2012. The primary end points were OS and radiographic progression-free survival (PFS). QoL was a prespecified exploratory end point. The trial was stopped after a planned interim analysis showed benefit of enzalutamide with fewer deaths compared to the placebo group (28\% vs 35\%, HR: 0.71, 95\% CI: [0.60-0.84]; $p<0.001$ ).

STRIVE, ${ }^{17}$ a Phase II trial, examined patients with CRPC and randomized them in a 1:1 ratio to receive enzalutamide vs bicalutamide $(50 \mathrm{mg}$ ) in addition to androgen deprivation therapy (ADT) in patients who had not previously been on chemotherapy. It recruited 396 patients between August 2012 and March 2014. The primary end point was PFS defined as no progression of soft tissue, bone, or PSA (with PSA progression defined by the PCWG3 definition). Of the 257 patients $(65 \%)$ with metastatic disease, the median PFS was 16.5 months in the enzalutamide group and 5.5 months in the bicalutamide group (HR: 0.24, 95\% CI: [0.17-0.34]; $p<0.001)$.

TERRAIN, a Phase III trial, enrolled 375 patients with mCRPC and randomized them in a 1:1 ratio to receive enzalutamide or bicalutamide $(50 \mathrm{mg})$ in addition to ADT. ${ }^{18}$ The trial recruited patients between March 2011 and July 2013. The primary end point was PFS defined as time from randomization to progression or death from any cause. The median PFS was 15.7 months with enzalutamide and 5.8 months with bicalutamide (HR: $0.44,95 \%$ CI: [0.34-0.57]; $p<0.0001$ ).

\section{Composite health outcomes}

The Functional Assessment of Cancer Therapy-Prostate (FACT-P) was the primary tool used in all four trials to assess patient-reported health-related QoL. The survey consists of the general FACT survey (FACT-G) and 12 prostate cancerspecific symptoms questions. ${ }^{19}$ The FACT-G consists of 27 questions scored on a $0-4$ Likert scale to assess the domains of physical, social and family, emotional, and functional wellbeing in an individual. All four trials evaluated FACT-P scores
(Table 2). These instruments have been examined for internal consistency, or how well each item measures the same underlying dimension, as expressed by the variable Cronbach's alpha. Commonly accepted ranges for Cronbach's alpha are as follows: $0.6-0.69$ for acceptable, $0.70-0.79$ for good, and $0.8-1.0$ for high. FACT-P has an alpha range of $0.87-0.89$.

The AFFIRM trial comparing enzalutamide to placebo in patients with mCRPC after chemotherapy collected FACT-P before randomization, on Weeks 13, 17, 21, 25, and every 12 weeks thereafter as long as they remained on their assigned arm of the study. ${ }^{1520}$ They defined a QoL response as a 10-point improvement on two consecutive measurements at least 3 weeks apart, a commonly accepted value for response in FACT-P. Of the 800 and 399 patients on the enzalutamide and placebo arms, $81 \%$ and $64 \%$ had FACT-P data included in the analysis, respectively. Mean baseline FACT-P scores were 108.7 in the enzalutamide group and 110.6 in the placebo group. There was a significant difference in QoL improvement of $43 \%$ vs $18 \%(p<0.001)$ in the enzalutamide arm as compared to the placebo arm during the study. FACT-P deterioration of ten points occurred in $47 \%$ of the enzalutamide group and $59 \%$ of the placebo group ( $p=0.001$ ). Median time to deterioration was 9 months in the enzalutamide group and 3.7 months in the placebo group $(p<0.0001)$.

PREVAIL, which compared enzalutamide to placebo in patients with MCRPC who had not yet received chemotherapy, scored FACT-P at baseline, Weeks 5, 13, and every 12 weeks until drug discontinuation. ${ }^{16,21}$ They defined a time to degradation of FACT-P as a 10-point decrease from baseline score, with no requirement for a consecutive measurement. Median baseline FACT-P scores were 121 for enzalutamide and 122 for placebo. Of all the patients enrolled in PREVAIL, 98\% in the enzalutamide group and $96 \%$ in the placebo group had adequate data for FACT-P analysis. There was a difference in QoL improvement in the enzalutamide $(40 \%)$ vs placebo $(23 \%)$ groups $(p<0.0001)$. The median time to deterioration was 11.3 months in the enzalutamide group and 5.6 months in the placebo group $(p<0.001)$.

In the STRIVE trial, ${ }^{17}$ which evaluated patients with CRPC on enzalutamide compared to bicalutamide, the median baseline FACT-P scores were 125.7 and 124, respectively. FACT-P decline was defined as time to a 10-point or greater decline in score, also with no requirement for a consecutive measurement. The trial did not report how often FACT-P was obtained or the percentage of patients who were included in the analysis. Median time to decline was 8.4 months in the enzalutamide group and 8.3 months in the bicalutamide group $(p=0.49)$. 
Table 2 Results of the most common patient-related outcome surveys on health-related quality of life and pain scores

\begin{tabular}{|c|c|c|c|c|c|}
\hline \multirow[t]{2}{*}{$\begin{array}{l}\text { Trial } \\
\end{array}$} & \multirow[t]{2}{*}{ Instrument } & \multirow[t]{2}{*}{ Survey frequency } & \multicolumn{2}{|c|}{ Response rate (response \% Response rate } & \multirow{2}{*}{$\begin{array}{l}\text { Median time to } \\
\text { deterioration }\end{array}$} \\
\hline & & & rate per arm) & (\% response rate per arm) & \\
\hline \multicolumn{6}{|l|}{ HRQoL } \\
\hline AFFIRM ${ }^{15}$ & FACT-P & $\begin{array}{l}\text { Before randomization, } \\
\text { Weeks } 13,17,21,25 \text {, and } \\
\text { every } 12 \text { weeks thereafter }\end{array}$ & $\begin{array}{l}938 / 1,199(674 / 800 \\
264 / 399)\end{array}$ & $78 \%(84 \%, 66 \%)$ & $\begin{array}{l}9 \text { months (enzalutamide) vs } \\
3.7 \text { months (placebo); } p<0.0001\end{array}$ \\
\hline \multirow[t]{2}{*}{ PREVAIL $^{16}$} & FACT-P & $\begin{array}{l}\text { Baseline, Weeks } 5,13 \text {, and } \\
\text { every } 12 \text { weeks thereafter }\end{array}$ & $\begin{array}{l}I, 669 / 1,717(856 / 872, \\
813 / 845)\end{array}$ & $97 \%(98 \%, 96 \%)$ & $\begin{array}{l}\text { II. } 3 \text { months (enzalutamide) vs } \\
5.6 \text { months (placebo); } p<0.00 \text { I }\end{array}$ \\
\hline & EQ-5D & $\begin{array}{l}\text { Baseline, Week } 13 \text { and } \\
\text { every } 12 \text { weeks thereafter }\end{array}$ & $\begin{array}{l}I, 448 / 1,717(818 / 872 \\
630 / 845)\end{array}$ & $84 \%(93 \%, 75 \%)$ & $\begin{array}{l}19.2 \text { months (enzalutamide) vs } \\
\text { II.I months (placebo); } p<0.000 \text { I }\end{array}$ \\
\hline STRIVE ${ }^{17}$ & FACT-P & Not reported & Not reported & Not reported & $\begin{array}{l}8.4 \text { months (enzalutamide) } \\
\text { vs } 8.3 \text { months (bicalutamide); } \\
p=0.49\end{array}$ \\
\hline TERRAIN $^{18}$ & ${ }^{3}$ FACT-P & Not reported & Not reported & Not reported & $\begin{array}{l}\text { I } 3.8 \text { months (enzalutamide) } \\
\text { vs } 8.5 \text { months (bicalutamide); } \\
p=0.026\end{array}$ \\
\hline \multicolumn{6}{|l|}{ Pain } \\
\hline & & & & & Pain progression at Week 13 \\
\hline AFFIRM ${ }^{15}$ & BPI-SF & $\begin{array}{l}\text { Baseline, Week } 13 \text { daily for } \\
7 \text { days }\end{array}$ & $\begin{array}{l}830 / 1,199(591 / 800 \\
239 / 399)\end{array}$ & $69 \%(74 \%, 60 \%)$ & $\begin{array}{l}28 \% \text { enzalutamide vs } 38 \% \\
\text { placebo }(p<0.0018)\end{array}$ \\
\hline PREVAIL $^{16}$ & BPI-SF & Baseline, Weeks 13 and 25 & $\begin{array}{l}1,379 / 1,717(769 / 872 \\
610 / 845)\end{array}$ & $80 \%(88 \%, 72 \%)$ & $\begin{array}{l}29 \% \text { enzalutamide vs } 42 \% \\
\text { placebo }(p<0.0001)\end{array}$ \\
\hline
\end{tabular}

Abbreviations: FACT-P, Functional Assessment of Cancer Therapy-Prostate; BPI-SF, Brief Pain Inventory - Short Form; HRQoL, health-related quality of life.

TERRAIN examined patients with mCRPC on enzalutamide compared to bicalutamide. ${ }^{18}$ Average FACT-P scores at randomization were 116.8 for the enzalutamide group and 118.3 for the bicalutamide group. A significant FACT-P change was defined as a 10-point deviation from baseline with no requirement for a repeat measurement. The frequency at which FACT-P was administered or the percentage of patients included in the calculations was not reported. The median time to deterioration was 13.8 months for the enzalutamide group vs 8.5 months for the bicalutamide group. Of the patients who filled out the survey, $33 \%$ in the enzalutamide group and $22 \%$ in the bicalutamide group showed improvement $(p=0.026)$.

In addition to the time to deterioration analysis, the AFFIRM researchers also used a longitudinal mixed model approach for FACT-P change, which showed a 1.5 point decrease in the enzalutamide group vs a 13.73 point decrease in the placebo group at 25 weeks $(p<0.001){ }^{22}$ A similar approach by the PREVAIL researchers using a longitudinal mixed model approach showed an average 5.08 point decrease in FACT-P in the enzalutamide group vs an average 10.8 point decrease in the placebo group at 61 weeks $(p<0.001){ }^{21}$

PREVAIL also had its participants fill out an EQ-5D, another standardized questionnaire for health status that can be used to calculate a quality-adjusted life year in cost-utility analyses. ${ }^{23}$ EQ-5D was collected at baseline, Week 13, and every 12 weeks thereafter until discontinuation of the study drug. EQ-5D analysis was performed on $94 \%$ of the patients on the enzalutamide arm and $75 \%$ on the placebo arm. There was an improvement in median time to EQ-5D deterioration in the enzalutamide arm compared to the placebo arm, 19.2 months vs 11.1 months, respectively $(p<0.0001)$.

\section{Pain}

Pain was assessed longitudinally with the Brief Pain Inventory - Short Form (BPI-SF) in three of the trials (AFFIRM, PREVAIL, and TERRAIN). BPI-SF is a widely used 11-point scale capturing pain intensity as well as interference with daily activity within the past 24 hours. ${ }^{24}$ The Cronbach's alpha for this form ranges from 0.80 to 0.92 , which is in the high internal consistency range.

In the AFFIRM trial, BPI-SF was administered daily for a week preceding the baseline and preceding the Week 13 visit. ${ }^{20}$ During randomization, patients were stratified by Eastern Cooperative Oncology Group (ECOG) and mean worst pain (0-3 vs 4-10) that week, the third question on the BPI-SF survey. Pain progression was defined by increase in average pain score at Week 13 compared with baseline and was assessed in 625 patients in the enzalutamide group and 259 patients in the placebo group. Fewer patients had pain progression in the enzalutamide group (28\%) than in the control group (38\%) $(p<0.0018)$.

In the PREVAIL trial, patients filled out the BPI-SF at baseline, Week 13, and Week 25. A clinically meaningful 
result was defined as either a $30 \%$ increase or two or more point increase from the baseline. The progression of pain at Week 13 was $29 \%$ in the enzalutamide group and $42 \%$ in the placebo group $(p<0.0001)$. At Week 25 , it was $32 \%$ in the enzalutamide group and $38 \%$ in the placebo group $(p=0.068)$.

The TERRAIN trial had patients fill out a BPI-SF survey at baseline and then at Week 49. Of the 375 patients who were enrolled, 97/184 patients in the enzalutamide group and $48 / 191$ patients in the bicalutamide group had data to compare. The average change in pain score was 0.83 (standard deviation 1.67) in the enzalutamide group and 1.05 (standard deviation 2.0) in the bicalutamide group.

\section{Adverse events}

The most common adverse events (AEs) seen in the AFFIRM trial were fatigue ( $34 \%$ vs $29 \%$ in enzalutamide vs placebo groups, respectively), diarrhea ( $21 \%$ vs $18 \%)$, hot flashes ( $20 \%$ vs $10 \%)$, musculoskeletal pain (14\% vs $10 \%)$, and headache $(12 \%$ vs $6 \%) .{ }^{15}$ Fatigue Grade 3 or higher was seen in $6 \%$ of patients on enzalutamide and $7 \%$ of patients on placebo. Discontinuation owing to an AE happened in $8 \%$ of patients on the enzalutamide arm and $10 \%$ of patients on the placebo arm.

The PREVAIL trial noted the most common AEs as fatigue ( $36 \%$ vs $26 \%$ in enzalutamide vs placebo), back pain ( $27 \%$ vs $22 \%$ ), constipation ( $22 \%$ vs $17 \%$ ), arthralgia ( $20 \%$ vs $16 \%$ ), and decreased appetite (18\% vs $16 \%)$. Hot flushes and diarrhea were also more common in the enzalutamide group as compared to the placebo group (18\% vs $8 \%$ and $16 \%$ vs $14 \%$, respectively). In addition, $13 \%$ developed hypertension in the enzalutamide arm vs $4 \%$ in the placebo arm.

STRIVE found the most common AEs were fatigue ( $38 \%$ vs $28 \%$ in enzalutamide vs bicalutamide), back pain (18\% vs $16 \%$ ), hot flashes (18\% vs $16 \%$ ), falls (14\% vs $8 \%$ ), and hypertension (12\% vs 5\%). Patients also experienced decreased appetite ( $12 \%$ vs $9 \%$ ), constipation ( $10 \%$ vs $17 \%$ ), and diarrhea ( $9 \%$ vs $14 \%$ ). Grade 3 or higher fatigue was experienced in nine patients taking enzalutamide and five patients taking bicalutamide. Grade 3 or higher hypertension was experienced in ten patients taking enzalutamide and three patients taking bicalutamide.

Patients in the TERRAIN trial most commonly reported fatigue ( $28 \%$ vs $20 \%$ in the enzalutamide vs bicalutamide groups), back pain (19\% vs 18\%), hot flashes (15\% vs $11 \%$ ), and nausea (14\% vs $17 \%$ ). Hypertension was seen in $14 \%$ of patients taking enzalutamide and $7 \%$ of patients taking bicalutamide. Constipation was seen in $12 \%$ of patients taking enzalutamide and $14 \%$ of patients taking bicalutamide. Arthralgias occurred in $10 \%$ of patients taking enzalutamide and $16 \%$ of patients taking bicalutamide. Grade 3 or higher hypertension occurred in 13 patients taking enzalutamide and eight patients taking bicalutamide.

\section{Serious AEs}

In the AFFIRM trial, five patients taking enzalutamide had seizures, whereas none on the placebo arm did. Both arms had two patients who had a myocardial infarction. In the PREVAIL trial, one patient in each arm had seizures. Seven patients taking enzalutamide had an acute cardiovascular event, whereas only four of the placebo group did. Twelve of the patients taking enzalutamide as compared to nine of the patients taking placebo had an ischemic or hemorrhagic cerebrovascular event. In the STRIVE trial, both the enzalutamide and the bicalutamide arms had six patients experience AEs leading to death. TERRAIN had five patients in the enzalutamide arm with myocardial infarction, whereas 0 in the bicalutamide arm. In addition, two people taking enzalutamide had seizures, as against one taking bicalutamide. There were nine deaths in the enzalutamide arm and three deaths in the bicalutamide arm. Deaths were due to fatal cardiac events $(n=3)$, and anemia, renal failure, cord compression, systemic inflammatory response syndrome, aspiration pneumonia, and paraneoplastic syndrome.

\section{Elderly patient populations}

Elderly patients were further analyzed in the AFFIRM and PREVAIL trials.

The AFFIRM trial performed a post hoc analysis on the patients aged 75 years and older, which consisted of 199 patients in the enzalutamide arm and 104 patients in the placebo arm. ${ }^{25}$ This group had a slightly higher ECOG performance status (ECOG $211 \%$ vs 8\%) than the younger patients, but otherwise baseline was well balanced. For those over 75 years, the median OS was 18.2 months in the enzalutamide group and 13.3 months in the placebo group (HR: 0.61, 95\% CI: [0.43-0.86]; $p=0.004$ ). The analysis did not comment on FACT-P or BPI-SF in the elderly. AEs occurred in this group included nausea (33\% vs 44\% in the enzalutamide group vs placebo group), fatigue (49\% vs $36 \%$ ), anorexia ( $28 \%$ vs $28 \%$ ), constipation ( $21 \%$ vs $28 \%$ ), and arthralgias (25\% vs $14 \%$ ). Two patients had seizures taking enzalutamide, whereas zero patients taking placebo had seizures.

In the PREVAIL trial, the analysis of patients aged 75 years or older was prespecified for the primary end points 
and for AEs. ${ }^{26}$ The elderly subgroup consisted of 609 patients (317 received enzalutamide and 292 received placebo) and had a similar distribution of disease, but were more likely to have $>20$ bone mets ( $22 \%$ vs $14 \%$ ). Elderly patients had a median OS of 32.4 months taking enzalutamide compared to 25.1 months taking placebo (HR: 0.61, 95\% CI: [0.47-0.79]; $p=0.0001$ ). Of the 609 patients, 295 of 317 in the enzalutamide group and 72 of 268 in the placebo group had data that could be analyzed. The median time to degradation of FACT-P was 10.9 months vs 5.5 months for the enzalutamide group as compared to the placebo group (HR: $0.73,95 \%$ CI: [0.57-0.92]). About $36 \%$ of the enzalutamide group and $27 \%$ of the placebo group had a clinically meaningful improvement (95\% CI: [1.4-16.7]). Of the 248 patients on the enzalutamide arm and the 135 patients on the placebo arm, pain progression at Week 25 by the BPI-SF occurred in $30.6 \%$ of the patients on enzalutamide and in $35 \%$ of the patients on placebo (95\% CI: [-14.0 to 5.7]). Common AEs in this population included fatigue (37.5\% vs $26 \%$ for enzalutamide and placebo, respectively), back pain $(27.4 \%$ vs $19.2 \%)$, constipation (23.7\% vs $17.5 \%)$, arthralgias (19.6\% vs $13.4 \%)$, decreased appetite (22.1\% vs $18.8 \%)$, hot flushes (15.5\% vs $6.8 \%$ ), diarrhea (18.6\% vs $15.8 \%$ ), and hypertension (11\% vs 3.4\%). Falls occurred in $19.2 \%$ of patients taking enzalutamide and $7.9 \%$ of patients taking placebo. AEs leading to death occurred in 17 patients taking enzalutamide and 23 patients taking placebo.

\section{Discussion}

Given the new therapies available for patients with advanced prostate cancer, it will be important to know the impact of these medications on PROs. Overall, in regard to enzalutamide in patients with mCRPC, the current studies suggest that they have favorable overall QoL scores compared to standard of care. This finding is also suggested in patients aged 75 years and older. Enzalutamide also seemed to show improved pain progression in the short term compared to placebo. The more frequently reported AEs included fatigue, back pain, and hot flashes. There were also a few patients who had adverse cardiovascular events or seizures.

There have been significant advances in methods for assessing PROs, and PCWG3 has laid out more specific recommendations for PROs as compared to its predecessor PCWG2. ${ }^{6,27}$ The group recommends reporting on clinically meaningful scores using previously validated instruments for outcomes such as time to deterioration of physical function and health-related QoL scores. For each reported outcome (eg, pain palliation), they recommend assessing for a clinically meaningful level (eg, 4 on a 10-point pain scale) at baseline with reassessment every 3-4 weeks, at treatment discontinuation, and 2-4 weeks later. Patient-reported pain has been studied the most in patients with prostate cancer, with an association with mortality, and PCWG3 additionally recommends serial scores over several days to get an accurate baseline, as pain scores can vary greatly over a week. They also suggest taking into account opioid use and assessment of pain interference with daily activities. ${ }^{28}$ If patient-reported symptoms from AEs are collected, the National Cancer Institute's patient-centered PRO-CTCAE assessment scale should be used. ${ }^{29}$

The AFFIRM trial had the most rigorous approach to analysis of PROs. The FACT-P deterioration cutoff required two consecutive questionnaires showing the same deterioration, and BPI-SF was obtained on consecutive days for a week to obtain more representative pain scores, consistent with PCWG3 guidelines. However, this also led to a lower survey completion rate compared to other trials. The impact of the $22 \%$ of patients who did not fill out the requisite number of FACT-P surveys or the $31 \%$ of patients who did not fill out adequate numbers of BPI-SF surveys is unknown. On the other hand, the PREVAIL trial did not require two consecutive surveys to demonstrate a significant QoL improvement or multiple pain surveys over time, which likely decreases the precision of the results. Finally, STRIVE and TERRAIN trials did not report how their QoL surveys were collected, at what intervals, and response rate, making it more challenging to draw conclusions regarding their results. In addition, only the AFFIRM, PREVAIL, and TERRAIN trials exclusively studied the population in question, those who have metastatic CRPC. For overall health-related QoL, AFFIRM, PREVAIL, and TERRAIN trials showed that enzalutamide compared favorably to placebo and low-dose bicalutamide. The STRIVE trial did not show a significant difference in median time to deterioration of the FACT-P. This may be because about one-third of the participants did not have metastatic disease while on the trial and thus may not have been as symptomatic as those with more advanced disease. Studies of patients with CRPC show a significant decline in QoL measurements, with pain being one of the most sensitive markers for deterioration. ${ }^{30}$ This may also explain why PREVAIL found a significant difference in pain progression at Week 13, but not at Week 25. Of the studies that looked at pain progression at Week 13, enzalutamide performed better than placebo.

Despite the additional recommendations set forth by the PCWG3 for reporting of PROs, there continues to be debate regarding how the data should be collected and analyzed. Systematic reviews of commonly used and validated 
health-related QoL instruments suggest that a more targeted questionnaire for patients with prostate cancer such as the University of California - Los Angeles Prostate Cancer Index (UCLA-PCI) or the Expanded Prostate Index Composite (EPIC) may provide more insight. ${ }^{31}$ In addition, there are currently no guidelines on how to report longitudinal changes, and some trials use a time to deterioration analysis, whereas others use a linear mixed model approach. ${ }^{32}$ Another limitation of these surveys is that PROs are secondary end points and all the four trials had a subset of patients who do not complete consecutive surveys, which can introduce attrition bias. Future trials will have to show more consistency in survey selection, frequency, and time points at which PROs are measured to yield more clinically useful information. In addition, these findings will have to be carefully applied to the real-world setting as patients in the community may have more symptomatic disease at baseline.

A similar agent used in the metastatic castrationresistant setting is the CYP-17 inhibitor and antiandrogen abiraterone. ${ }^{33}$ Currently, there are no completed head-tohead trials comparing enzalutamide with any other agents. In trials of patients with MCRPC with or without prior chemo, abiraterone plus prednisone has been shown to decrease FACT-P degradation compared with prednisone alone. ${ }^{34-38}$ In the COU-AA-301 trial, patients with mCRPC who had previously received chemotherapy were randomized to abiraterone or placebo, which showed improved OS in a preplanned interim analysis (median OS: 14.8 months vs 10.9 months; HR: 0.65, 95\% CI: [0.54-0.77]; $p=0.001$ ). The median time to FACT-P degradation was 13.8 months vs 8.3 months for the abiraterone plus prednisone vs prednisone-alone group, respectively (HR: 0.607, 95\% CI: [0.495-0.743]; $p=0.0001)$. In the COU-AA-302 trial, patients with $\mathrm{mCRPC}$ were randomized to abiraterone plus prednisone or prednisone alone prior to chemotherapy, and they also showed improved OS (median OS 34.7 months vs 30.3 months; HR: $0.81,95 \%$ CI: [0.7-0.93]; $p=0.003$ ). In this trial, the median time to FACT-P degradation was 12.7 months in the abiraterone plus prednisone group and 8.3 months in the prednisone only group (HR: $0.78,95 \% \mathrm{CI}$ : [0.66-0.92]; $p=0.003)$. Other agents that are currently being tested in the $\mathrm{MCRPC}$ population include the second-generation antiandrogen ODM-201 (NCT02799602), and potentially in the future, antiandrogen apalutamide (ARN-509). There are several studies that will examine enzalutamide compared with combinations including chemotherapy, zoledronic acid, radiation, and ADT that are actively recruiting including NCT00268476 (STAMPEDE), NCT02254785, and NCT02677896.

\section{Disclosure}

Julie N Graff has received research funding and an honorarium from Astellas Pharma, Inc. The authors report no other conflicts of interest in this work.

\section{References}

1. Siegel RL, Miller KD, Jemal A. Cancer statistics, 2016. CA Cancer J Clin. 2016;66(1):7-30.

2. Torre LA, Bray F, Siegel RL, Ferlay J, Lortet-Tieulent J, Jemal A. Global cancer statistics, 2012. CA Cancer J Clin. 2015;65(2):87-108.

3. Huggins C, Hodges CV. Studies on prostatic cancer: I. The effect of castration, of estrogen and of androgen injection on serum phosphatases in metastatic carcinoma of the prostate. Cancer Res. 1941;1(1):293-297. Available from: http://www.ncbi.nlm.nih.gov/pubmed/12050481.

4. Crawford ED, Eisenberger MA, McLeod DG, Spaulding JT, Benson R. A controlled trial of leuprolide with and without flutamide in prostatic carcinoma. N Engl J Med. 1989;321(7):419-424.

5. Akaza H. Combined androgen blockade for prostate cancer: review of efficacy, safety and cost-effectiveness. Cancer Sci. 2011;102(1):51-56.

6. Scher HI, Morris MJ, Stadler WM, et al. Trial design and objectives for castration-resistant prostate cancer: updated recommendations from the prostate cancer clinical trials working Group 3.J Clin Oncol. 2016;34(12):1402-1418.

7. Kluetz PG, Ning YM, Maher VE, et al. Abiraterone acetate in combination with prednisone for the treatment of patients with metastatic castration-resistant prostate cancer: U.S. food and drug administration drug approval summary. Clin Cancer Res. 2013;19(24):6650-6656.

8. Ning YM, Brave M, Maher VE, et al. U.S. Food and drug administration approval summary: enzalutamide for the treatment of patients with chemotherapy-naive metastatic castration-resistant prostate cancer. Oncologist. 2015;20(8):960-966.

9. Mohler J, Armstrong A, Bahnson R, et al. Prostate cancer. NCCN Guidel. 2016;1-96. Available from: http://www.nccn.org/professionals/physician_gls/pdf/prostate.pdf. Accessed November 22, 2015.

10. Tran C, Ouk S, Clegg NJ, et al. Development of a second-generation antiandrogen for treatment of advanced prostate cancer. 2009; 324(5928):787-790.

11. Bambury RM, Scher HI. Enzalutamide: development from bench to bedside. Urol Oncol. 2015;33(6):280-288.

12. Astellas Pharma US I. XTANDI (Enzalutamide) Oral Capsules, 2012. Available from: http://www.accessdata.fda.gov/.

13. Basch $\mathrm{E}$. The missing voice of patients in drug-safety reporting. NEJM. 2010;363(1):1-3.

14. Basch E, Deal AM, Kris MG, et al. Symptom monitoring with patientreported outcomes during routine cancer treatment: a randomized controlled trial. J Clin Oncol. 2016;34(6):557-565.

15. Scher HI, Fizazi K, Saad F, et al. Increased survival with enzalutamide in prostate cancer after chemotherapy. $N$ Engl J Med. 2012;367(13): 1187-1197.

16. Beer TM, Armstrong AJ, Rathkopf DE, et al. Enzalutamide in metastatic prostate cancer before chemotherapy. N Engl J Med. 2014; 371(5):424-433.

17. Penson DF, Armstrong AJ, Concepcion R, et al. Enzalutamide versus bicalutamide in castration-resistant prostate cancer: the STRIVE trial. J Clin Oncol. 2016.

18. Shore ND, Chowdhury S, Villers A, et al. Efficacy and safety of enzalutamide versus bicalutamide for patients with metastatic prostate cancer (TERRAIN): a randomised, double-blind, phase 2 study. Lancet Oncol. 2016;2045(15):1-11.

19. Esper P, Mo F, Chodak G, Sinner M, Cella D, Pienta KJ. Measuring quality of life in men with prostate cancer using the functional assessment of cancer therapy-prostate instrument. Urology. 1997;50(97):920-928.

20. Fizazi K, Scher HI, Miller K, et al. Effect of enzalutamide on time to first skeletal-related event, pain, and quality of life in men with castrationresistant prostate cancer: results from the randomised, phase 3 AFFIRM trial. Lancet Oncol. 2014;15(10):1147-1156. 
21. Loriot Y, Miller K, Sternberg CN, et al. Effect of enzalutamide on health-related quality of life, pain, and skeletal-related events in asymptomatic and minimally symptomatic, chemotherapy-naive patients with metastatic castration-resistant prostate cancer (PREVAIL): results from a randomised, phase 3 trial. Lancet Oncol. 2015;16(5):509-521.

22. Cella D, Ivanescu C, Holmstrom S, Bui CN, Spalding J, Fizazi K. Impact of enzalutamide on quality of life in men with metastatic castrationresistant prostate cancer after chemotherapy: additional analyses from the AFFIRM randomized clinical trial. Ann Oncol. 2015;26(1):179-185.

23. Rabin R, de Charro F. EQ-5D: a measure of health status from the EuroQol Group. Ann Med. 2001;33(5):337-343. Available from: http:// www.ncbi.nlm.nih.gov/pubmed/11491192. Accessed July 8, 2016.

24. Cleeland $\mathrm{C}$. The brief pain inventory user guide. Br Pain Invent. 2009;3-4. Available from: http://www.mdanderson.org/education-andresearch/departments-programs-and-labs/departments-and-divisions/ symptom-research/symptom-assessment-tools/BPI_UserGuide.pdf.

25. Sternberg CN, de Bono JS, Chi KN, et al. Improved outcomes in elderly patients with metastatic castration-resistant prostate cancer treated with the androgen receptor inhibitor enzalutamide: results from the phase III AFFIRM trial. Ann Oncol. 2014;25(2):429-434.

26. Graff JN, Baciarello G, Armstrong AJ, et al. Efficacy and safety of enzalutamide in patients 75 years or older with chemotherapy-naive metastatic castration-resistant prostate cancer: results from PREVAIL. Ann Oncol. 2016;27(2):286-294.

27. Chen RC, Chang P, Vetter RJ, et al. Recommended patient-reported core set of symptoms to measure in prostate cancer treatment trials. J Natl Cancer Inst. 2014;106(7):1-7.

28. Halabi S, Vogelzang NJ, Kornblith AB, et al. Pain predicts overall survival in men with metastatic castration-refractory prostate cancer. J Clin Oncol. 2008;26(15):2544-2549.

29. Basch E, Reeve BB, Mitchell SA, et al. Development of the National Cancer Institute's patient-reported outcomes version of the common terminology criteria for adverse events (PRO-CTCAE). J Natl Cancer Inst. 2014;106(9).
30. Kirby M, Hirst C, Crawford ED. Characterising the castration-resistant prostate cancer population: a systematic review. Int J Clin Pract. 2011; 65(11):1180-1192.

31. Hamoen EH, De Rooij M, Witjes JA, Barentsz JO, Rovers MM. Measuring health-related quality of life in men with prostate cancer: a systematic review of the most used questionnaires and their validity. Urol Oncol. 2015;33(2):e19-e28.

32. Bonnetain F, Fiteni F, Efficace F, Anota A. Statistical challenges in the analysis of health-related quality of life in cancer clinical trials. J Clin Oncol. 2016;34(16):1-6.

33. Attard G, Reid AHM, Auchus RJ, et al. Clinical and biochemical consequences of CYP17A1 inhibition with abiraterone given with and without exogenous glucocorticoids in castrate men with advanced prostate cancer. J Clin Endocrinol Metab. 2012;97(2):507-516.

34. de Bono JS, Logothetis CJ, Molina A, et al. Abiraterone and increased survival in metastatic prostate cancer. $N$ Engl J Med. 2011;364(21): 1995-2198.

35. Basch E, Autio K, Ryan CJ, et al. Abiraterone acetate plus prednisone versus prednisone alone in chemotherapy-naive men with metastatic castration-resistant prostate cancer: patient-reported outcome results of a randomised phase 3 trial. Lancet Oncol. 2013;14(12):1193-1199.

36. Ryan CJ, Smith MR, Fizazi K, et al. Abiraterone acetate plus prednisone versus placebo plus prednisone in chemotherapy-naive men with metastatic castration-resistant prostate cancer (COU-AA-302): final overall survival analysis of a randomised, double-blind, placebo-controlled phase 3 study. Lancet Oncol. 2015;16(2):152-160.

37. Rathkopf DE, Smith MR, De Bono JS, et al. Updated interim efficacy analysis and long-term safety of abiraterone acetate in metastatic castration-resistant prostate cancer patients without prior chemotherapy (COU-AA-302). Eur Urol. 2014;66(5):815-825.

38. Harland S, Staffurth J, Molina A, et al. Effect of abiraterone acetate treatment on the quality of life of patients with metastatic castrationresistant prostate cancer after failure of docetaxel chemotherapy. Eur $J$ Cancer. 2013;49(17):3648-3657.
Research and Reports in Urology

\section{Publish your work in this journal}

Research and Reports in Urology is an international, peer-reviewed, open access journal publishing original research, reports, editorials, reviews and commentaries on all aspects of adult and pediatric urology in the clinic and laboratory including the following topics: Pathology, pathophysiology of urological disease; Investigation and treatment of

\section{Dovepress}

urological disease; Pharmacology of drugs used for the treatment of urological disease. The manuscript management system is completely online and includes a very quick and fair peer-review system, which is all easy to use. Visit http://www.dovepress.com/testimonials.php to read real quotes from published authors. 